\title{
Global Realization of Strongly Pseudoconvex CR Manifolds
}

Dedicated to Professor S. Nakano on his 60th birthday

By

\section{Takeo OHSAWA*}

\section{Introduction}

Let $S$ be a real hypersurface in a complex manifold $M$. Then, $T_{M}^{1,0}$, the holomorphic tangent bundle of $M$, determines an integrable subbundle $T_{S}^{\prime}:=\left.T_{M}^{1.0}\right|_{s} \cap\left(T_{S} \otimes \mathbf{C}\right) \subset T_{S} \otimes \mathbf{C}$. Modelled on $\left(S, T_{S}^{\prime}\right)$, a $C R$ manifold (a Cauchy-Riemann manifold) is defined as a pair $\left(X, T_{X}^{\prime}\right)$ consisting of a differentiable manifold $X$ and a subbundle $T_{X}^{\prime}$ of $T_{X} \otimes \mathrm{C}$ satisfying the following two conditions:

(i) $\quad T_{X}^{\prime} \cap \bar{T}_{X}^{\prime}=0$,

(ii) $\quad T_{X}^{\prime}$ is closed under the Poisson bracket (integrability condition).

Newlander-Nirenberg's theorem [9] says that a $C R$ manifold is nothing but a complex manifold if $\operatorname{dim} X=\operatorname{rank}_{\mathbf{R}} T_{X}^{\prime}$. Thus, an interesting problem arises concerning the realizability, or imbeddability, of $G R$ manifolds as submanifolds of complex manifolds. Boutet de Monvel [1] showed that s. p.c. manifolds (cf. Section 1) are holomorphically imbeddable into some $\mathbf{C}^{N}$, provided that $\operatorname{dim} X \geqq 5$. Recently Kuranishi [7] proved that locally every s. p. c. manifold is imbeddable as a real hypersurface of the ball in $\mathbf{C}^{n}$, provided that $\operatorname{dim} X \geqq 9$.

Our result is as follows.

Theorem. Let $\left(X, T_{X}^{\prime}\right)$ be a compact s.p.c. manifold of dimension $\geqq 5$. Then, $\left(X, T_{X}^{\prime}\right)$ is realizable as a hypersurface of a complex manifold.

Received May 28, 1983.

* Research Institute for Mathematical Sciences, Kyoto University, Kyoto 606, Japan. 
Our proof is quite different from Kuranishi's argument and based upon Boutet de Monvel's imbedding theorem which assures the existence of a realization $X \subset \mathbf{C}^{N}$ for sufficiently large $N$. We apply then Tanaka's stability theorem in [10] to perform a finite number of bumps on $X$ and obtain an s.p.c. manifold $\hat{X}$ which is the boundary of a complex manifold containing $X$ as a hypersurface.

The author is very grateful to Professor S. Nakano for his interest in the problem. He also thanks the referee and Professor N. Tanaka for pointing out mistakes.

\section{Preliminaries}

We recall briefly Boutet de Monvel's imbedding theorem and Tanaka's stability theorem.

Let $\left(X, T_{X}^{\prime}\right)$ be a compact $C R$ manifold of dimension $2 n-1$, and let $T_{X} \otimes \mathbf{C}=T_{X}^{\prime} \oplus \bar{T}_{X}^{\prime} \oplus F$ be a decomposition with $F=\bar{F}$.

Definition 1. $\left(X, T_{X}^{\prime}\right)$ is called a strongly pseudoconvex $C R$ manifold, or shortly an s. p. c. manifold, if $\operatorname{rank}_{\mathrm{c}} F=1$ and for any local frame $\left\{v_{1}, \cdots, v_{n-1}\right\}$ of $T_{X}^{\prime}$, we can choose a local frame $\{\theta\}$ of $F$ with $\bar{\theta}=\theta$ such that the $(n-1) \times(n-1)$ matrix $\left(c_{i j}\right)$ defined by $\sqrt{-1} c_{i j} \theta \equiv\left[v_{i}, v_{j}\right]\left(\bmod T_{X}^{\prime} \oplus \bar{T}_{X}^{\prime}\right)$ is positive definite.

We note that the above condition is satisfied by a real hypersurface $S$ in a complex manifold $M$ if and only if for any point $p \in S$ there exist a neighbourhood $U$ in $M$ and a $C^{\infty}$ function $\varphi$ on $U$ such that $S \cap U=\{\varphi=0\}, d \varphi \neq 0$, and $\partial \bar{\partial} \varphi>0$.

A $\mathbf{C}$-valued function $f$ defined on an open set $V \subset X$ is said to be holomorphic if $f$ is of class $C^{\infty}$ and $\bar{v} f=0$ for any section $v$ of $T_{X}^{\prime}$ over $V$. We denote by $\mathcal{O}_{X}$ the sheaf over $X$ of the germs of holomorphic functions.

Theorem 1. Let $\left(X, T_{X}^{\prime}\right)$ be an s.p.c. manifold of dimension $2 n-1$. If $n \geqq 3$, there exist an integer $N$ and holomorphic functions $f_{i}, i=1,2$, $\cdots, N$, such that the map $F:=\left(f_{1}, \cdots, f_{N}\right)$ gives a $C^{\infty}$ imbedding of $X$ into $\mathbf{C}^{N}$.

Proof. See Boutet de Monvel [1]. 
Corollary 1. Every s.p.c. manifold of dimension $\geqq 5$ is isomorphic to a CR submanifold of a complex number space.

Proof. Let $F: X \hookrightarrow \mathrm{C}^{N}$ be an imbedding by holomorphic functions. Then, for any point $x \in X, F_{*}\left(T_{X, x}^{\prime}\right) \subset T_{\mathrm{c}^{N, F(x)}}^{1,0} \cap\left(T_{F(X)} \otimes \mathbf{C}\right)$. Since rank $T_{X}^{\prime}=n-1$, this inclusion is an equality.

Corollary 2. Every s.p.c. manifold of dimension $\geqq 5$ is locally realizable as a hypersurface.

Proof. Immediate from Corollary 1.

Definition 2. A complex manifold with boundary $\bar{M}$ is a $C^{\infty}$ manifold with boundary of dimension $2 n$ with a system of coordinate patches

$$
\psi_{i}: U_{i} \widetilde{\Im}\left\{\left.\left(z_{1}, \cdots, z_{n}\right) \in \mathbf{C}^{n}\left|\sum_{k=1}^{n}\right| z_{k}\right|^{2}<1, r_{i}\left(z_{1}, \cdots, z_{n}\right) \geqq 0\right\},
$$

where $r_{i}$ is a $C^{\infty}$ real valued function on the ball with $d r \neq 0$ everywhere, such that $\phi_{j} \psi_{i}^{-1}$ is holomorphic on $\phi_{\imath}\left(U_{i} \cap U_{j}\right) \backslash\left\{r_{i}=0\right\}$.

We denote by $\partial M$ the boundary of $\bar{M}$ and put $\partial M=\bar{M} \backslash M$. Clearly, $\partial M$ has a canonical structure of $C R$-manifold.

Proposition 2. Let $X \subset \mathbf{C}^{N}$ be a compact $(2 n-1)$-dimensional $C^{\infty}$ submanifold. Suppose $X$ is s.p.c. with respect to the induced CR structure and that $n \geqq 2$. Then, there exists a unique analytic subvariety $W$ in $\mathbf{C}^{N} \backslash X$ whose closure $\bar{W}$ in $\mathbf{C}^{N}$ is compact and satisfies $\bar{W} \backslash W=X$. Moreover, Sing $W$, the set of singular points of $W$, consists of a finite set of points and $\bar{W} \backslash$ Sing $W$ is a complex manifold with boundary.

Proof. The reader is referred to Kuranishi [12], section 2.

In virtue of Hironaka's desingularization theorem [4], we obtain from $\bar{W}$, by a finite succession of blowing ups, a complex manifold with boundary $\bar{M}$. We shall call $\bar{M}$ the associated complex manifold of $X$.

Let $C^{0, q}(\bar{M})$ denote the $C^{\infty}(0, q)$-forms on $\bar{M}$ and let $C_{0}^{0, q}(\bar{M}):=$ $\left\{f \in C^{0, q}(\bar{M}) ;\left.f\right|_{\partial M}=0\right\}$. We set 


$$
\begin{aligned}
& Z^{0, q}(\bar{M})=\left\{f \in C^{0, q}(\bar{M}) ; \bar{\partial} f=0\right\}, \\
& B^{0, q}(\bar{M})=\bar{\partial} C^{0, q-1}(\bar{M}) . \\
& H^{0, q}(\bar{M})=Z^{0, q}(\bar{M}) / B^{0, q}(\bar{M}), \\
& Z_{0}^{0, q}(\bar{M})=\left\{f \in Z^{0, q}(\bar{M}) ;\left.f\right|_{X}=0\right\} \\
& B_{0}^{0, q}(\bar{M})=\bar{\partial} C_{0}^{0, q-1}(\bar{M}) \cap C_{0}^{0, q}(\bar{M}), \\
& H_{0}^{0, q}(\bar{M})=Z_{0}^{0, q}(\bar{M}) / B_{0}^{0, q}(\bar{M}) .
\end{aligned}
$$

Let $C^{0, q}(X)$ denote the $C^{\infty}$ sections of the bundle $\wedge^{q} \bar{T}_{X}^{\prime *} \longrightarrow X$. We set

$$
\begin{aligned}
Z^{0, q}(X)= & \left\{f \in C^{0, q}(X) ;\langle d f, v\rangle=0 \text { for } v \in \bigwedge^{q \perp 1} \bar{T}_{X}^{*}\right\}, \\
B^{0, q}(X)= & \left\{f \in C^{0, q}(X) ;\right. \\
& \left.f=\wedge^{q} \bar{T}_{X}^{\prime *} \text {-part of } d g, \text { for some } g \in C^{0, q-1}(X)\right\},
\end{aligned}
$$

and

$$
H^{0, q}(X)=Z^{0, q}(X) / B^{0, q}(X) .
$$

We shall be allowed simply to refer [11] and [2] concerning the properties of $H^{0, q}(\bar{M}), H_{0}^{0, q}(\bar{M})$, and $H^{0, q}(X)$. Namely we have, under the situation that $\partial \bar{M}=X$,

Proposition 3 (cf. [2] and [11] Proposition 6.6).

$$
H_{0}^{0, q}(\bar{M})=0, \text { for } q \leqq n-1 \text {. }
$$

Corollary 3. $\quad H^{0, q}(\bar{M}) \cong H^{0, q}(X)$ if $q<n-1$.

Similarly as in [5], Theorem 3.4.8, we have

Proposition 4. $H^{0, q}(\bar{M}) \cong H^{0, q}(M)$, for $q \geqq 1$.

Definition 3. A family $\left\{X_{t}\right\}_{t \in T}$ of $C R$ manifolds is called a differentiable family if there exist a $C R$ manifold $\mathscr{X}$, a $C^{\infty}$ manifold $T$ and a proper surjective smooth map $\pi: \mathscr{X} \rightarrow T$ such that $\left(X_{t}, T_{X_{t}}^{\prime}\right)$ $\cong\left(\pi^{-1}(t), T_{X}^{\prime} \cap\left(T_{\pi^{-1}(t)} \otimes \mathbf{C}\right)\right)$.

We quote here Tanaka's stability theorem for holomorphic imbeddings.

Theorem 2. Let $\left\{X_{t}\right\}_{t \in T}$ be a differentiable family of s.p.c. manifolds satisfying the following conditions. 
(i) $\quad \operatorname{dim} X_{t} \geqq 5$

(ii) $\operatorname{dim} H^{0,1}\left(X_{t}\right)$ does not depend on $t$.

Then, for any $t_{0} \in T$ and holomorphic imbedding $F_{0}: X_{t_{0}} \longrightarrow \mathrm{C}^{N}$, there exist a neighbourhood $T^{\prime} \ni t_{0}$ and a $C^{\infty}$ map $\mathscr{F}: \cup_{t \in T^{\prime}} X_{t} \longrightarrow \mathbb{C}^{N}$ such that $\left.\mathscr{F}\right|_{x_{t_{0}}}=F_{0}$ and that $\left.F\right|_{X_{t}}$ are holomorphic imbeddings for $t \in T^{\prime}$.

Proof. See [10], Theorem 9.4.

\section{Bumping of S.P.C. Manifolds}

Let $X$ be an s. p. c. manifold of dimension $2 n-1 \geqq 5$, let $x \in X$ be any point, and let $B(r)$ be the ball $\left\{\left.\left(z_{1}, \cdots, z_{n}\right) \in \mathbf{C}^{n}\left|\sum_{k=1}^{n}\right| z_{k}\right|^{2}<r\right\}$. Then, by Corollary 2, there exist a neighbourhood $U \ni x$ and an imbedding $F: U \hookrightarrow B(1)$ by holomorphic functions. $F(U)$ is then defined by an equation $\varphi=0$ on $B(1 / 2)$, where $\varphi$ is of class $C^{\infty}, d \varphi \neq 0$ and $\partial \bar{\partial} \varphi>0$.

Let $\rho$ be a nonnegative $C^{\infty}$ function on $B(1 / 2)$ such that $\operatorname{supp} \rho$ $\subset B(1 / 4)$ and $\rho=1$ on $B(1 / 8)$. Then, for sufficiently small $\varepsilon>0$, we have $\partial \bar{\partial}(\varphi-t \rho)>0$ and $d(\varphi-t \rho) \neq 0$, for any $t \in[-\varepsilon, \varepsilon]$. Choose a $C^{\infty}$ family of imbeddings $\tilde{F}: U \times[-\varepsilon, \varepsilon] \rightarrow B(1)$ such that

(*) $\quad \tilde{F}(x, t)=F(x)$ for $x \in F^{-1}(B(1) \backslash B(1 / 4))$ and $t \in[-\varepsilon, \varepsilon]$. (**) $\quad \tilde{F}(U \times\{t\}) \cap B(1 / 2)=\{\varphi-t \rho=0\}$.

Using $\tilde{F}$, we define a $C R$ structure $T_{\mathscr{X}}^{\prime}$ on $\mathscr{X}=X \times(-\varepsilon, \varepsilon)$ as follows:

Let $p: X \times(-\varepsilon, \varepsilon) \longrightarrow X$ and $\pi: X \times(-\varepsilon, \varepsilon) \longrightarrow(-\varepsilon, \varepsilon)$ be the projections. We put $T_{\mathscr{X},(x, t)}^{\prime}=p^{*} T_{X, x}^{\prime}$ for $x \in X \backslash U$, and $T_{\mathscr{X},(x, t)}^{\prime}=$ $\tilde{F}_{t *}^{-1}\left(T_{\tilde{F}(U \times\{t\}), \tilde{F}(x, t)}^{\prime}\right)$ for $x \in U$, where $\tilde{F}_{t}=\tilde{F}(, t)$. Then we have a differentiable family $\left\{X_{t}\right\}$ of s. p. c. manifolds defined by $\left(\mathscr{X}, T_{\mathscr{X}}^{\prime}\right)$ and $\pi: \mathscr{X} \longrightarrow(-\varepsilon, \varepsilon)$. We shall call it a bumping family of $X$ with front $U$. Let $\bar{M}_{0}$ be a complex manifold with boundary such that $\partial M_{0} \cong X_{0}$. Then, there exist a neighbourhood $W \supset F(U)$ in $B(1)$ and a holomorphic imbedding $\tau: W \cap\{\varphi \leqq 0\} \subseteq \bar{M}_{0}$ such that $\tau=F^{-1}$ on $F(U)$. Thus, for sufficiently small $t>0$, we can patch $\bar{M}_{0}$ and $W \cap\{\varphi-t \rho \leqq 0\}$ via the identification $\tau(W \cap\{\varphi \leqq 0\}) \cong W \cap\{\varphi \leqq 0\} \subset$ $W \cap\{\varphi-t \rho<0\}$, and obtain complex manifolds with boundary $\bar{M}_{t}$ such that $\partial M_{t} \cong X_{t}$. On the other hand, for $t \leqq 0, \bar{M}_{t}:=\bar{M}_{0} \backslash\{\varphi-t \rho>0\}$ 
are complex manifolds with boundary, if $|t|$ is sufficiently small, and we have $\partial M_{t} \cong X_{t}$, too. Fix $\varepsilon$ so that we have a family of compact complex manifolds with boundary $\left\{\bar{M}_{t}\right\}$ with $\partial M_{t} \cong X_{t}$ for $|t|<\varepsilon$.

Let $A$ be the maximal compact analytic set of $M_{0}$, then we have $H^{q}\left(M_{0}, \mathcal{O}_{M_{0}}\right) \cong H^{q}\left(A, \mathcal{O}_{M_{0} \mid A}\right)$ for $q \geqq 1$. (cf. Narasimhan [8]). From the definition of $M_{t}$, choosing $\varepsilon$ smaller if necessary we may assume that the maximal compact analytic sets of $M_{t}$ and their neighbourhoods are analytically equivalent for all $t$. Hence we can suppose $H^{q}\left(M_{0}\right.$, $\left.\mathcal{O}_{M_{0}}\right) \cong H^{q}\left(M_{t}, \mathcal{O}_{M_{t}}\right)$ for $q \geqq 1$. Thus, combining Proposition 4 and Corollary 3 with this fact, we have

Proposition 5. $H^{0,1}\left(X_{t}\right) \cong H^{0,1}(X)$ for $: t \mid<\varepsilon$.

\section{Proof of Theorem}

We shall now prove that every compact s.p.c. manifold of dimension $\geqq 5$ is realizable as a hypersurface of a complex manifold. Let the notations be as in Section 2. By Theorem 1 we may assume that $X$ is a $C R$ submanifold of $\mathbf{C}^{N}$. We take a finite covering $\left\{V_{i}\right\}_{i=1}^{m}$ of $X$ by open sets of $\mathbf{C}^{N}$ and systems of affine coordinates $\left(z_{i 1}, \cdots, z_{i N}\right)$ of $\mathbf{C}^{N}$ so that the projections $p_{i}:\left(z_{i 1}, \cdots, z_{i N}\right) \longrightarrow\left(z_{i 1}, \cdots\right.$, $z_{i n}$ ) define holomorphic imbeddings $\varphi_{i}$ of $\overline{V_{i} \cap X}$ into $\overline{B(1)} \subset \mathbf{C}^{n}$. We may assume moreover that $\cup \varphi_{i}^{-1}(B(1 / 8))=X$. Let $\left\{X_{t}\right\}$ be a bumping family of $X$ with front $V_{1} \cap X$. Then, by Proposition 5, we can apply Theorem 2 and obtain a differentiable family of holomorphic imbeddings $\Phi_{1}: \underset{-\delta<t<\delta}{\cup} X_{t} \longrightarrow \mathbf{C}^{N}$ for sufficiently small $\delta$. Thus, there exist $t_{1}>0$ such that $\cup p_{i}{ }^{-1}(B(1 / 8)) \cap V_{i} \supset \Phi_{1}\left(X_{t_{1}}\right)$ and that $p_{i}$ are one-to-one immersions on neighbourhoods in $\Phi_{1}\left(X_{t_{1}}\right)$ of $\overline{V_{i} \cap \Phi_{1}\left(X_{t_{1}}\right)}$. Let $\left\{\bar{M}_{t}\right\}$ be the corresponding family of complex manifolds with boundary. Then we have $\bar{M}_{t_{1}} \supset X$. Inductively we can define $\Phi_{k}$ and obtain a $C R$ manifold $X_{t_{1} \cdots t_{k}}$ as a member of a bumping family of $X_{t_{1} \cdots t_{k-1}}$ with front $\Phi_{k-1}^{-1}\left(V_{k} \cap \Phi_{k-1}\left(X_{t_{1} \cdots t_{k-1}}\right)\right)$. Here, $\mathrm{t}_{a}>0, a=1,2$, $\cdots, k$. Let $\bar{M}_{t_{1} \cdots t_{k}}$ be the complex manifold with boundary corresponding to $X_{t_{1} \cdots t_{k}}$. Clearly $M_{t_{1} \cdots t_{m}} \supset X$ for sufficiently small $t_{1}, \cdots, \mathrm{t}_{m}$.

Q. E. D. 


\section{References}

[1] Boutet de Monvel, L., Intégration des équations de Cauchy-Riemann induites formelles, Séminaire Goulaouic-Lions-Schwartz 1974-75.

[2] Grauert, H. and Riemenschneider, O., Kählersche Mannigfaltigkeiten mit hyper-qkonvexem Rand, Problems in Analysis, N. J., Princeton Univ. Press 1970. 61-79.

[3] Gunning, R. C. and Rossi, H., Analytic functions of several complex variables, Englewood Cliffs, N. J., Prentice Hall Inc., 1965.

[4] Hironaka, H., Resolution of singularities of an algebraic variety over a field of characteristic zero I, II, Ann. of Math., 79 (1964) 109-326.

[5] Hörmander, L., $L^{2}$ estimates and existence theorems for the $\bar{\partial}$ operator, Acta Math., 113 (1965), 89-152.

[6] - An introduction to complex analysis in several variables, Van Nostrand, Princeton, N. J., 1966.

[7] Kuranishi, M., Strongly pseudoconvex CR structures over small balls, Part III. An embedding theorem, Ann. of Math. 116 (1982) 249-330.

[8] Narasimhan R., The Levi problem for complex spaces II, Math. Ann. 146, (1962) 195-216.

[9] Newlander, A. and Nirenberg, L., Complex analytic coordinates in almost-complex manifolds, Ann. of Math., 65 (1957) 391-404.

[10] Tanaka, N., A differential geometric study on strongly pseudoconvex manifolds, Lectures in mathematics, Kyoto Univ. 9.

[11] Kohn, J. J. and Rossi, H., On the extension of holomorphic functions from the boundary of a complex manifold, Ann. of Math. 81 (1965) 451-472.

[12] Kuranishi, M., Application of $\bar{\partial}_{b}$ to deformation of isolated singularities Proc. of Symp. in Pure Math., 30 AMS, (1977), 97-106. 
